Pacific

Journal of

Mathematics

\title{
ON STABLE SOLUTIONS OF THE BIHARMONIC PROBLEM WITH POLYNOMIAL GROWTH
}

Hatem Hajlaoui, Abdellaziz Harrabi and Dong Ye 


\title{
ON STABLE SOLUTIONS OF THE BIHARMONIC PROBLEM WITH POLYNOMIAL GROWTH
}

\author{
Hatem Hajlaoui, AbDellaziz Harrabi And Dong Ye
}

We prove the nonexistence of smooth stable solutions to the biharmonic problem $\Delta^{2} u=u^{p}, u>0$ in $\mathbb{R}^{N}$ for $1<p<\infty$ and $N<2\left(1+x_{0}\right)$, where $x_{0}$ is the largest root of the equation

$$
x^{4}-\frac{32 p(p+1)}{(p-1)^{2}} x^{2}+\frac{32 p(p+1)(p+3)}{(p-1)^{3}} x-\frac{64 p(p+1)^{2}}{(p-1)^{4}}=0 .
$$

In particular, as $x_{0}>5$ when $p>1$, we obtain the nonexistence of smooth stable solutions for any $N \leq 12$ and $p>1$. Moreover, we consider also the corresponding problem in the half-space $\mathbb{R}_{+}^{N}$, and the elliptic problem $\Delta^{2} u=\lambda(u+1)^{p}$ on a bounded smooth domain $\Omega$ with the Navier boundary conditions. We prove the regularity of the extremal solution in lower dimensions.

\section{Introduction}

Consider the biharmonic equation

$$
\Delta^{2} u=u^{p}, \quad u>0 \quad \text { in } \mathbb{R}^{N}
$$

where $N \geq 5$ and $p>1$. Let

$$
\Lambda(\phi):=\int_{\mathbb{R}^{N}}|\Delta \phi|^{2} d x-p \int_{\mathbb{R}^{N}} u^{p-1} \phi^{2} d x \quad \text { for all } \phi \in H^{2}\left(\mathbb{R}^{N}\right) .
$$

A solution $u$ is said to be stable if $\Lambda(\phi) \geq 0$ for any test function $\phi \in H^{2}\left(\mathbb{R}^{N}\right)$.

In this note, we prove the following classification result.

Theorem 1.1. Let $N \geq 5$ and $p>1$. Equation (1-1) has no classical stable solution if $N<2+2 x_{0}$, where $x_{0}$ is the largest root of the polynomial

$$
H(x)=x^{4}-\frac{32 p(p+1)}{(p-1)^{2}} x^{2}+\frac{32 p(p+1)(p+3)}{(p-1)^{3}} x-\frac{64 p(p+1)^{2}}{(p-1)^{4}} .
$$

Moreover, we have $x_{0}>5$ for any $p>1$. Consequently, if $N \leq 12$, (1-1) has no classical stable solution for all $p>1$. 
For the corresponding second-order problem,

$$
\Delta u+|u|^{p-1} u=0 \quad \text { in } \mathbb{R}^{N}, \quad p>1,
$$

Farina has obtained the optimal Liouville type result for all finite Morse index solutions. He proved in [Farina 2007] that a smooth finite Morse index solution to (1-4) exists if and only if $p \geq p_{J L}$ and $N \geq 11$, or $p=\frac{N+2}{N-2}$ and $N \geq 3$. Here $p_{J L}$ is the so-called Joseph-Lundgren exponent; see (1.11) in [Gui et al. 1992].

The nonexistence of positive solutions to (1-1) is shown if $p<\frac{N+4}{N-4}$, and all entire solutions are classified if $p=\frac{N+4}{N-4}$; see [Lin 1998; Wei and Xu 1999]. On the other hand, the radially symmetric solutions to (1-1) are studied in [Ferrero et al. 2009; Gazzola and Grunau 2006; Guo and Wei 2010; Karageorgis 2009]. In particular, Karageorgis [2009] proved that the radial entire solution to (1-1) is stable if and only if $p \geq p_{J L_{4}}$ and $N \geq 13$. Here $p_{J L_{4}}$ stands for the corresponding Joseph-Lundgren exponent to $\Delta^{2}$.

The general fourth-order case (1-1) is more delicate, since the integration by parts argument used by Farina cannot be adapted easily. The first nonexistence result for general stable solutions was proved by Wei and Ye [2013], who proposed we consider (1-1) as a system

$$
-\Delta u=v, \quad-\Delta v=u^{p} \quad \text { in } \mathbb{R}^{N},
$$

and introduced the idea to use different test functions with $u$ but also $v$. Using estimates in [Souplet 2009] they showed that for $N \leq 8$, (1-1) has no smooth stable solutions. For $N \geq 9$, using a blow-up argument, they proved that the classification holds still for $p<N /(N-8)+\epsilon_{N}$ with $\epsilon_{N}>0$, but without any explicit value of $\epsilon_{N}$. This result was improved by Wei, Xu and Yang in [Wei et al. 2013] for $N \geq 20$ with a more explicit bound.

Using the stability of system (1-5) and an interesting iteration argument, Cowan [2013, Theorem 2] proved that there is no smooth stable solution to (1-1) if $N<$ $2+\frac{4(p+1)}{p-1} t_{0}$, where

$$
t_{0}=\sqrt{\frac{2 p}{p+1}}+\sqrt{\frac{2 p}{p+1}-\sqrt{\frac{2 p}{p+1}}} \text { for all } p>1 .
$$

In particular, if $N \leq 10,(1-1)$ has no stable solution for any $p>1$.

However, the study for radial solutions in [Karageorgis 2009] suggests the following conjecture.

Conjecture. A smooth stable solution to (1-1) exists if and only if $p \geq p_{J L_{4}}$ and $N \geq 13$.

Consequently, the Liouville type result for stable solutions of (1-1) should hold true for $N \leq 12$ with any $p>1$; that's what we prove here. More precisely, by 
[Karageorgis 2009, Theorem 1], the radial entire solutions to (1-1) are unstable if and only if

$$
\frac{N^{2}(N-4)^{2}}{16}<p Q_{4}\left(-\frac{4}{p-1}\right),
$$

where $Q_{4}(m)=m(m-2)(m+N-2)(m+N-4)$. The left-hand side comes from the best constant of the Hardy-Rellich inequality (see [Rellich 1969]): Let $N \geq 5$,

$$
\int_{\mathbb{R}^{N}}|\Delta \varphi|^{2} d x \geq \frac{N^{2}(N-4)^{2}}{16} \int_{\mathbb{R}^{N}} \frac{\varphi^{2}}{|x|^{4}} d x \quad \text { for all } \varphi \in H^{2}\left(\mathbb{R}^{N}\right) .
$$

The right-hand side of (1-7) comes from the weak radial solution $w(x)=|x|^{-4 /(p-1)}$. When $p>\frac{N+4}{N-4}$, we can check that $w \in H_{\text {loc }}^{2}\left(\mathbb{R}^{N}\right)$ and

$$
\Delta^{2} w=Q_{4}\left(-\frac{4}{p-1}\right) w^{p} \quad \text { in } \mathscr{D}^{\prime}\left(\mathbb{R}^{N}\right) .
$$

Since $w^{p-1}(x)=|x|^{-4}$, and in view of the Hardy-Rellich inequality, the condition (1-7) means just that $w$ is not a stable solution in $\mathbb{R}^{N}$, that is, there exists $\varphi \in H^{2}\left(\mathbb{R}^{N}\right)$ such that

$$
\Lambda_{w}(\varphi):=\int_{\mathbb{R}^{N}}|\Delta \varphi|^{2} d x-p \int_{\mathbb{R}^{N}} Q_{4}\left(-\frac{4}{p-1}\right) w^{p-1} \varphi^{2} d x<0 .
$$

If we set $N=2+2 x$, a direct calculation shows that (1-7) is equivalent to $H_{J L_{4}}(x)<$ 0 , where

$$
H_{J L_{4}}(x):=\left(x^{2}-1\right)^{2}-\frac{32 p(p+1)}{(p-1)^{2}} x^{2}+\frac{32 p(p+1)(p+3)}{(p-1)^{3}} x-\frac{64 p(p+1)^{2}}{(p-1)^{4}} .
$$

By [Gazzola and Grunau 2006], (1-7) is equivalent to $N<2+2 x_{1}$ if $x_{1}$ denotes the largest root of $H_{J L_{4}}$. Note that closeness between the fourth-order polynomials $H_{J L_{4}}$ and $H$ (in Theorem 1.1); they differ only by $H(x)-H_{J L_{4}}(x)=2 x^{2}-1$.

Furthermore, Theorem 1.1 improves the bound given in [Cowan 2013] for all $p>1$. Indeed, Lemmas 2.2 and 2.4 below imply that $x_{0}>\frac{2(p+1)}{p-1} t_{0}$.

Recall that to handle the equation (1-1), we prove in general that $v=-\Delta u>0$ in $\mathbb{R}^{N}$ by studying function averages on the sphere; see [Wei and Xu 1999]. Applying the blow-up argument as in [Souplet 2009; Wei and Ye 2013], we can assume that $u$ and $v$ are uniformly bounded in $\mathbb{R}^{N}$. Therefore the following Souplet's estimate [2009] holds true in $\mathbb{R}^{N}$, which was established for any bounded solution $u$ of (1-1):

$$
v \geq \sqrt{\frac{2}{p+1}} u^{(p+1) / 2} .
$$

Here we propose a new approach. Without assuming the boundedness of $u$ or showing immediately the positivity of $v$, we prove first some integral estimates for 
stable solutions of (1-1), which will enable us the estimate (1-8). This idea permits us to handle more general biharmonic equations: let $N \geq 5$ and $p>1$, and consider

$$
\Delta^{2} u=u^{p}, \quad u>0 \text { in } \Sigma \subset \mathbb{R}^{N}, \quad u=\Delta u=0 \text { on } \partial \Sigma .
$$

Let $E=H^{2}(\Sigma) \cap H_{0}^{1}(\Sigma)$ and

$$
\Lambda_{0}(\phi):=\int_{\Sigma}|\Delta \phi|^{2} d x-p \int_{\Sigma} u^{p-1} \phi^{2} d x \quad \text { for all } \phi \in E .
$$

A solution $u$ of (1-9) is said to be stable if $\Lambda_{0}(\phi) \geq 0$ for any $\phi \in E$.

Proposition 1.2. Let $u$ be a classical stable solution of (1-9) where $\Sigma$ is one of $\mathbb{R}^{N}$, the half-space $\Sigma=\mathbb{R}_{+}^{N}$, or the exterior domain $\Sigma=\mathbb{R}^{N} \backslash \bar{\Omega}$ or $\mathbb{R}_{+}^{N} \backslash \bar{\Omega}$, where $\Omega$ is a bounded smooth domain of $\mathbb{R}^{N}$. Then the inequality (1-8) holds in $\Sigma$, and consequently $v>0$ in $\Sigma$.

Using this, we obtain a Liouville type result for (1-9) in the half-space situation, which improves the result in [Wei and Ye 2013] for a wider range of $N$, and without assuming the boundedness of $u$ or $v=-\Delta u$.

Theorem 1.3. Let $x_{0}$ be defined as in Theorem 1.1. If $N<2+2 x_{0}$, there exists no classical stable solution of (1-9) if $\Sigma=\mathbb{R}_{+}^{N}$.

Our proof combines also many ideas from [Wei and Ye 2013; Cowan and Ghoussoub 2014; Cowan 2013]. Briefly, for (1-1), we apply different test functions to both equations of the system (1-5) and make use of the following inequality in [Cowan and Ghoussoub 2014] (see also [Cowan 2013; Dupaigne et al. 2013a]): if $u$ is a stable solution of $(1-1)$, then

$$
\int_{\mathbb{R}^{N}} \sqrt{p} u^{(p-1) / 2} \varphi^{2} d x \leq \int_{\mathbb{R}^{N}}|\nabla \varphi|^{2} d x \quad \text { for all } \varphi \in C_{0}^{1}\left(\mathbb{R}^{N}\right) .
$$

This will enable us to make two estimates. From these estimates, we prove that for any stable solution $u$ of $(1-1), \phi \in C_{0}^{2}\left(\mathbb{R}^{N}\right)$ and $s \geq 1$,

$$
L(s)<0 \Rightarrow \int_{\mathbb{R}^{N}} u^{p} v^{s-1} \phi^{2} d x \leq C \int_{\mathbb{R}^{N}} v^{s}\left(\left|\Delta\left(\phi^{2}\right)\right|+|\nabla \phi|^{2}\right) d x .
$$

Here $L$ is a polynomial of degree 4, see (2-9) below, and the constant $C$ depends only on $p$ and $s$. Applying then the iteration argument of Cowan [2013], we show that $u \equiv 0$ if $N<2+2 x_{0}$, which is a contradiction, since $u$ is positive.

Using similar ideas, we consider the elliptic equation on bounded domains:

$$
\begin{cases}\Delta^{2} u=\lambda(u+1)^{p} & \text { in a bounded smooth domain } \Omega \subset \mathbb{R}^{N}, N \geq 1 \\ u=\Delta u=0 & \text { on } \partial \Omega .\end{cases}
$$

It is well known (see [Berchio and Gazzola 2005; Gazzola et al. 2010]) that there exists a critical value $\lambda^{*}>0$ depending on $p>1$ and $\Omega$ such that: 
- If $\lambda \in\left(0, \lambda^{*}\right),\left(P_{\lambda}\right)$ has a minimal and classical solution $u_{\lambda}$ which is stable.

- If $\lambda=\lambda^{*}$, then $u^{*}=\lim _{\lambda \rightarrow \lambda^{*}} u_{\lambda}$ is a weak solution to $\left(P_{\lambda^{*}}\right) ; u^{*}$ is called the extremal solution.

- No solution of $\left(P_{\lambda}\right)$ exists whenever $\lambda>\lambda^{*}$.

In [Cowan et al. 2010; Wei and Ye 2013], it was proved that if $1<p<((N-$ $8) / N)_{+}^{-1}$, or equivalently $N<8 p /(p-1)$, the extremal solution $u^{*}$ is smooth. Recently, Cowan and Ghoussoub improved the above result by showing that $u^{*}$ is smooth if $N<2+4(p+1) /(p-1) t_{0}$ with $t_{0}$ in (1-6), so $u^{*}$ is smooth for any $p>1$ when $N \leq 10$. Our result is this:

Theorem 1.4. The extremal solution $u^{*}$ is smooth if $N<2+2 x_{0}$ with $x_{0}$ given by Theorem 1.1. In particular, $u^{*}$ is smooth for any $p>1$ if $N \leq 12$.

We remark that our proof does not use the a priori estimate of $v=-\Delta u$ as in [Cowan et al. 2010; Cowan and Ghoussoub 2014].

The paper is organized as follows. We prove some preliminary results and Proposition 1.2 in Section 2. The proofs of Theorems 1.1, 1.3 and 1.4 are given in Sections 3 and 4.

\section{Preliminaries}

We show first how to obtain the estimate (1-8) for stable solutions of (1-9). Our idea is to use the stability condition (1-10) to get some decay estimates for stable solutions of (1-9). In the following, we denote by $B_{r}$ the ball of center 0 and radius $r>0$.

Lemma 2.1. Let $u$ be a stable solution to (1-9) and set $v=-\Delta u$. Then

$$
\int_{\Sigma \cap B_{R}}\left(v^{2}+u^{p+1}\right) d x \leq C R^{N-4-8 /(p-1)} \text { for all } R>0 .
$$

Proof. We proceed similarly as in Step 1 of the proof for [Wei and Ye 2013, Theorem 1.1], but we do not assume here that $v>0$ or $u$ is bounded in $\Sigma$. For any $\xi \in C^{4}(\Sigma)$ satisfying $\xi=\Delta \xi=0$ on $\partial \Sigma$ and $\eta \in C_{0}^{\infty}\left(\mathbb{R}^{N}\right)$, we have

$$
\begin{aligned}
\int_{\Sigma}\left(\Delta^{2} \xi\right) \xi \eta^{2} d x=\int_{\Sigma}[\Delta(\xi \eta)]^{2} d x+ & \int_{\Sigma}\left[-4(\nabla \xi \cdot \nabla \eta)^{2}+2 \xi \Delta \xi|\nabla \eta|^{2}\right] d x \\
& +\int_{\Sigma} \xi^{2}\left[2 \nabla(\Delta \eta) \cdot \nabla \eta+(\Delta \eta)^{2}\right] d x
\end{aligned}
$$

The proof is direct as in [Wei and Ye 2013, Lemma 2.3], noticing just that in the integrations by parts, all boundary integration terms on $\partial \Sigma$ vanish under the Navier conditions for $\xi$. 
Let $u$ be a solution of (1-9). Taking $\xi=u$ in (2-2), we have

$$
\begin{aligned}
& \int_{\Sigma}[\Delta(u \eta)]^{2} d x-\int_{\Sigma} u^{p+1} \eta^{2} d x \\
& \quad=4 \int_{\Sigma}(\nabla u \nabla \eta)^{2} d x+2 \int_{\Sigma} u v|\nabla \eta|^{2} d x-\int_{\Sigma} u^{2}\left[2 \nabla(\Delta \eta) \cdot \nabla \eta+(\Delta \eta)^{2}\right] d x,
\end{aligned}
$$

where $v=-\Delta u$. Using $\phi=u \eta$ in (1-10), we obtain easily

$$
\begin{aligned}
& \int_{\Sigma}\left[(\Delta(u \eta))^{2}+u^{p+1} \eta^{2}\right] d x \\
& \leq C_{1} \int_{\Sigma}\left[|\nabla u|^{2}|\nabla \eta|^{2}+u^{2}|\nabla(\Delta \eta) \cdot \nabla \eta|+u^{2}(\Delta \eta)^{2}\right] d x+C_{2} \int_{\Sigma} u v|\nabla \eta|^{2} d x .
\end{aligned}
$$

Here and below, $C$ and $C_{i}$ denote generic positive constants independent of $u$, which can change from one line to another. Since $\Delta(u \eta)=2 \nabla u \cdot \nabla \eta+u \Delta \eta-v \eta$ we get from (2-3)

$$
\begin{aligned}
& \int_{\Sigma}\left[v^{2} \eta^{2}+u^{p+1} \eta^{2}\right] d x \\
& \leq C_{1} \int_{\Sigma}\left[|\nabla u|^{2}|\nabla \eta|^{2}+u^{2}|\nabla(\Delta \eta) \cdot \nabla \eta|+u^{2}(\Delta \eta)^{2}\right] d x+C_{2} \int_{\Sigma} u v|\nabla \eta|^{2} d x .
\end{aligned}
$$

On the other hand, since $u=0$ on $\partial \Sigma$,

$$
\begin{aligned}
2 \int_{\Sigma}|\nabla u|^{2}|\nabla \eta|^{2} d x & =\int_{\Sigma} \Delta\left(u^{2}\right)|\nabla \eta|^{2} d x+2 \int_{\Sigma} u v|\nabla \eta|^{2} d x \\
& =\int_{\Sigma} u^{2} \Delta\left(|\nabla \eta|^{2}\right) d x+2 \int_{\Sigma} u v|\nabla \eta|^{2} d x .
\end{aligned}
$$

By inputting this into (2-4), we arrive at

$$
\begin{aligned}
& \int_{\Sigma}\left[v^{2} \eta^{2}+u^{p+1} \eta^{2}\right] d x \\
& \leq C_{1} \int_{\Sigma} u^{2}\left[|\nabla(\Delta \eta) \cdot \nabla \eta|+(\Delta \eta)^{2}+\left|\Delta\left(|\nabla \eta|^{2}\right)\right|\right] d x+C_{2} \int_{\Sigma} u v|\nabla \eta|^{2} d x .
\end{aligned}
$$

If we let $\eta=\varphi^{m}$ with $m>2$ and $\varphi \in C_{0}^{\infty}\left(\mathbb{R}^{N}\right), \varphi \geq 0$, it follows that

$$
\begin{aligned}
\int_{\Sigma} u v|\nabla \eta|^{2} d x & =m^{2} \int_{\Sigma} u v \varphi^{2(m-1)}|\nabla \varphi|^{2} d x \\
& \leq \frac{1}{2 C} \int_{\Sigma}\left(v \varphi^{m}\right)^{2} d x+C \int_{\Sigma} u^{2} \varphi^{2(m-2)}|\nabla \varphi|^{4} d x .
\end{aligned}
$$

Now choose a cutoff function $\varphi_{0}$ in $C_{0}^{\infty}\left(B_{2}\right)$ satisfying $0 \leq \varphi_{0} \leq 1$ and $\varphi_{0}=1$ for $|x|<1$. Inputting the above inequality into (2-5) with $\varphi=\varphi_{0}\left(R^{-1} x\right)$ for $R>0$ and 
$\eta=\varphi^{m}$ with $m=(2 p+2) /(p-1)>2$, we arrive at

$$
\begin{aligned}
\int_{\Sigma}\left(v^{2}+u^{p+1}\right) \varphi^{2 m} d x & \leq \frac{C}{R^{4}} \int_{\Sigma} u^{2} \varphi^{2 m-4} d x \\
& \leq \frac{C}{R^{4}}\left(\int_{\Sigma} u^{p+1} \varphi^{(p+1)(m-2)} d x\right)^{2 /(p+1)} R^{N(p-1) /(p+1)} \\
& =\frac{C}{R^{4}}\left(\int_{\Sigma} u^{p+1} \varphi^{2 m} d x\right)^{2 /(p+1)} R^{N(p-1) /(p+1)} .
\end{aligned}
$$

Hence

$$
\int_{\Sigma} u^{p+1} \varphi^{2 m} d x \leq C R^{N-4(p+1) /(p-1)} .
$$

Combining with (2-6) we get (2-1), since $\varphi^{2 m}=1$ for $x \in B_{R}:=\left\{x \in \mathbb{R}^{N}:|x| \leq R\right\}$.

Proof of Proposition 1.2. Let

$$
\zeta=\beta u^{(p+1) / 2}-v, \quad \text { where } \beta=\sqrt{\frac{2}{p+1}} .
$$

Then a direct computation shows that $\Delta \zeta \geq \beta^{-1} u^{(p-1) / 2} \zeta$ in $\Sigma$. Consider $\zeta_{+}:=$ $\max (\zeta, 0)$. For any $R>0$, we have

$$
\begin{aligned}
\int_{\Sigma \cap B_{R}}\left|\nabla \zeta_{+}\right|^{2} d x & =-\int_{\Sigma \cap B_{R}} \zeta_{+} \Delta \zeta d x+\int_{\partial\left(\Sigma \cap B_{R}\right)} \zeta_{+} \frac{\partial \zeta}{\partial v} d \sigma \\
& \leq \int_{\Sigma \cap \partial B_{R}} \zeta_{+} \frac{\partial \zeta}{\partial v} d \sigma .
\end{aligned}
$$

Here we used $\zeta_{+} \Delta \zeta \geq 0$ in $\Sigma$ and $\zeta=0$ on $\partial \Sigma$. Now let $S^{N-1}$ denote the unit sphere in $\mathbb{R}^{N}$ and

$$
e(r)=\int_{S^{N-1} \cap\left(r^{-1} \Sigma\right)} \zeta_{+}^{2}(r \sigma) d \sigma \quad \text { for } r>0 .
$$

We remark that there exists an $R_{0}>0$ satisfying

$$
\int_{\Sigma \cap \partial B_{r}} \zeta_{+} \frac{\partial \zeta}{\partial \nu} d \sigma=\frac{r^{N-1}}{2} e^{\prime}(r) \quad \text { for all } r \geq R_{0} .
$$

Moreover, for $R \geq R_{0}$, we deduce from (2-1) that

$$
\begin{aligned}
\int_{R_{0}}^{R} r^{N-1} e(r) d r & \leq \int_{B_{R} \cap \Sigma} \zeta_{+}^{2} d x \leq C \int_{B_{R} \cap \Sigma}\left(v^{2}+u^{p+1}\right) d x \\
& \leq C R^{N-4-8 /(p-1)}=o\left(R^{N}\right) .
\end{aligned}
$$

This means that the function $e$ cannot be nondecreasing at infinity, so there exists a sequence $R_{j} \rightarrow \infty$ satisfying $e^{\prime}\left(R_{j}\right) \leq 0$. Combining (2-7) and (2-8) with 
$R=R_{j} \rightarrow \infty$, we obtain

$$
\int_{\Sigma}\left|\nabla \zeta_{+}\right|^{2} d x=0
$$

Using $\zeta=0$ on $\partial \Sigma$, we have $\zeta_{+} \equiv 0$ in $\Sigma$, or equivalently (1-8) holds true in $\Sigma$. Clearly $v>0$ in $\Sigma$ by (1-8).

In the following, we show some properties of the polynomials $L$ and $H$, useful for our proofs. Let

$$
L(s)=s^{4}-32 \frac{p}{p+1} s^{2}+32 \frac{p(p+3)}{(p+1)^{2}} s-64 \frac{p}{(p+1)^{2}}, \quad s \in \mathbb{R} .
$$

Lemma 2.2. $L\left(2 t_{0}\right)<0$ and $L$ has a unique root $s_{0}$ in the interval $\left(2 t_{0}, \infty\right)$. Proof. Obviously

$$
L\left(2 t_{0}\right)=16 t_{0}^{4}-128 \frac{p}{p+1} t_{0}^{2}+64 \frac{p(p+3)}{(p+1)^{2}} t_{0}-64 \frac{p}{(p+1)^{2}} .
$$
Since $t_{0}^{2} /\left(2 t_{0}-1\right)=\sqrt{2 p /(p+1)}\left(\right.$ see [Cowan 2013]), we have $t_{0}^{4}=\frac{2 p}{p+1}\left(2 t_{0}-1\right)^{2}$.
A direct computation yields

$$
\begin{aligned}
\frac{(p+1)^{2} L\left(2 t_{0}\right)}{32 p} & =(p+1)\left(2 t_{0}-1\right)^{2}-4(p+1) t_{0}^{2}+2(p+3) t_{0}-2 \\
& =(p-1)\left(1-2 t_{0}\right) .
\end{aligned}
$$

Since $t_{0}>1$ for any $p>1$, we have $L\left(2 t_{0}\right)<0$. Furthermore, for all $p>1, s \geq 2 t_{0}$, we have

$$
\begin{aligned}
(p+1) L^{\prime \prime}(s)=12(p+1) s^{2}-64 p & \geq 48(p+1) t_{0}^{2}-64 p \\
& \geq 48(p+1) \frac{2 p}{p+1}-64 p=32 p>0
\end{aligned}
$$

in $\left[2 t_{0}, \infty\right)$, where we used $t_{0}^{2} \geq 2 p /(p+1)$, which holds by (1-6). Therefore $L$ is convex in $\left[2 t_{0}, \infty\right)$. Since $\lim _{s \rightarrow \infty} L(s)=\infty$ and $L\left(2 t_{0}\right)<0$, it's clear that $L$ admits a unique root in $\left(2 t_{0}, \infty\right)$.

Remark 2.3. After the change of variable $x=\frac{p+1}{p-1} s$, a direct calculation gives

$$
H(x)=\left(\frac{p+1}{p-1}\right)^{4} L(s),
$$

hence $H(x)<0$ if and only if $L(s)<0$. Using the lemma above, we see that $x_{0}=\frac{p+1}{p-1} s_{0}$ is the largest root of $H$, and $x_{0}$ is the only root of $H$ for $x \geq \frac{2(p+1)}{p-1} t_{0}$.

Lemma 2.4. If $x_{0}=\frac{p+1}{p-1} s_{0}$ is the largest root of $H$, then $x_{0}>5$ for any $p>1$. 
Proof. Since $x_{0}$ is the largest root of $H$, to have $x_{0}>5$ it suffices to show $H(5)<0$. Let $J(p)=(p-1)^{4} H(5)$; then $J(p)=-15 p^{4}-1284 p^{3}+4262 p^{2}-3844 p+625$. Therefore,

$J^{\prime}(p)=-60 p^{3}-3852 p^{2}+8524 p-3844, \quad J^{\prime \prime}(p)=-180 p^{2}-7704 p+8524$.

We see that $J^{\prime \prime}<0$ in $[2, \infty)$. Consequently $J^{\prime}(p)<0$ and $J(p)<0$ for $p \geq 2$. Hence $x_{0}>5$ if $p \geq 2$. For $p \in(1,2)$, we have $x_{0}>\frac{2(p+1)}{p-1} t_{0} \geq 6 t_{0}$, which exceeds 5 since $t_{0}>1$.

\section{Proof of Theorems 1.1 and 1.3}

We will prove only Theorem 1.1, since the proof of Theorem 1.3 is completely similar, just changing $B_{r}$ to $B_{r} \cap \mathbb{R}_{+}^{N}$.

The following result generalizes [Cowan 2013, Lemma 4], which is a crucial argument for our proof. As above, the constant $C$ always denotes a positive number which may change term by term, but does not depend on the solution $u$. For $k \in \mathbb{N}$, let $R_{k}:=2^{k} R$ with $R>0$.

Lemma 3.1. Assume that $u$ is a classical stable solution of (1-1). Then for all $2 \leq s<s_{0}$, there is $C<\infty$ such that

$$
\int_{B_{R_{k}}} u^{p} v^{s-1} d x \leq \frac{C}{R^{2}} \int_{B_{R_{k+1}}} v^{s} d x \quad \text { for all } R>0 .
$$

Proof. Let $u$ be a classical stable solution of (1-1). Let $\phi \in C_{0}^{2}\left(\mathbb{R}^{N}\right)$ and $\varphi=$ $u^{(q+1) / 2} \phi$ with $q \geq 1$. With this $\varphi$, the stability inequality (1-11) gives

$$
\begin{aligned}
& \sqrt{p} \int_{\mathbb{R}^{N}} u^{(p-1) / 2} u^{q+1} \phi^{2} \\
& \quad \leq \int_{\mathbb{R}^{N}} u^{q+1}|\nabla \phi|^{2}+\int_{\mathbb{R}^{N}}\left|\nabla u^{(q+1) / 2}\right|^{2} \phi^{2}+(q+1) \int_{\mathbb{R}^{N}} u^{q} \phi \nabla u \nabla \phi .
\end{aligned}
$$

Integrating by parts, we get

$$
\begin{aligned}
\int_{\mathbb{R}^{N}}\left|\nabla u^{\frac{q+1}{2}}\right|^{2} \phi^{2} d x & =\frac{(q+1)^{2}}{4} \int_{\mathbb{R}^{N}} u^{q-1}|\nabla u|^{2} \phi^{2} d x \\
& =\frac{(q+1)^{2}}{4 q} \int_{\mathbb{R}^{N}} \phi^{2} \nabla\left(u^{q}\right) \nabla u d x \\
& =\frac{(q+1)^{2}}{4 q} \int_{\mathbb{R}^{N}} u^{q} v \phi^{2} d x-\frac{q+1}{4 q} \int_{\mathbb{R}^{N}} \nabla\left(u^{q+1}\right) \nabla\left(\phi^{2}\right) d x \\
& =\frac{(q+1)^{2}}{4 q} \int_{\mathbb{R}^{N}} u^{q} v \phi^{2} d x+\frac{q+1}{4 q} \int_{\mathbb{R}^{N}} u^{q+1} \Delta\left(\phi^{2}\right) d x
\end{aligned}
$$


and

$$
\begin{aligned}
(q+1) \int_{\mathbb{R}^{N}} u^{q} \phi \nabla u \nabla \phi d x & =\frac{1}{2} \int_{\mathbb{R}^{N}} \nabla\left(u^{q+1}\right) \nabla\left(\phi^{2}\right) d x \\
& =-\frac{1}{2} \int_{\mathbb{R}^{N}} u^{q+1} \Delta\left(\phi^{2}\right) d x .
\end{aligned}
$$

Combining (3-2)-(3-4), we conclude that

$$
a_{1} \int_{\mathbb{R}^{N}} u^{(p-1) / 2} u^{q+1} \phi^{2} d x \leq \int_{\mathbb{R}^{N}} u^{q} v \phi^{2} d x+C \int_{\mathbb{R}^{N}} u^{q+1}\left(\left|\Delta\left(\phi^{2}\right)\right|+|\nabla \phi|^{2}\right) d x
$$

where $a_{1}=(4 q \sqrt{p}) /(q+1)^{2}$. Now choose $\phi(x)=h\left(R_{k}^{-1} x\right)$, where $h \in C_{0}^{\infty}\left(B_{2}\right)$ is such that $h \equiv 1$ in $B_{1}$. Then

$$
\int_{\mathbb{R}^{N}} u^{(p-1) / 2} u^{q+1} \phi^{2} d x \leq \frac{1}{a_{1}} \int_{\mathbb{R}^{N}} u^{q} v \phi^{2} d x+\frac{C}{R^{2}} \int_{B_{R_{k+1}}} u^{q+1} d x .
$$

Now, apply the stability inequality (1-11) with $\varphi=v^{(r+1) / 2} \phi, r \geq 1$, to obtain

$$
\begin{aligned}
\sqrt{p} \int_{\mathbb{R}^{N}} u^{(p-1) / 2} v^{r+1} \phi^{2} & \\
& \leq \int_{\mathbb{R}^{N}} v^{r+1}|\nabla \phi|^{2}+\int_{\mathbb{R}^{N}}\left|\nabla v^{(r+1) / 2}\right|^{2} \phi^{2}+(r+1) \int_{\mathbb{R}^{N}} v^{r} \phi \nabla v \nabla \phi .
\end{aligned}
$$

By a very similar computation (recalling that $-\Delta v=u^{p}$ ), we have

$$
\int_{\mathbb{R}^{N}} u^{(p-1) / 2} v^{r+1} \phi^{2} d x \leq \frac{1}{a_{2}} \int_{\mathbb{R}^{N}} u^{p} v^{r} \phi^{2} d x+\frac{C}{R^{2}} \int_{B_{R_{k+1}}} v^{r+1} d x
$$

where $a_{2}=(4 r \sqrt{p}) /(r+1)^{2}$.

Using (3-6) and (3-7), we get

$$
\begin{aligned}
I_{1}+a_{2}^{r+1} I_{2} & :=\int_{\mathbb{R}^{N}} u^{(p-1) / 2} u^{q+1} \phi^{2} d x+a_{2}^{r+1} \int_{\mathbb{R}^{N}} u^{(p-1) / 2} v^{r+1} \phi^{2} d x \\
\leq & \frac{1}{a_{1}} \int_{\mathbb{R}^{N}} u^{q} v \phi^{2} d x+a_{2}^{r} \int_{\mathbb{R}^{N}} u^{p} v^{r} \phi^{2} d x \\
& +\frac{C}{R^{2}} \int_{B_{R_{k+1}}}\left(u^{q+1}+v^{r+1}\right) d x .
\end{aligned}
$$

Now fix

$$
2 q=(p+1) r+p-1, \quad \text { or equivalently } \quad q+1=\frac{1}{2}(p+1)(r+1) .
$$


By Young's inequality, we get

$$
\begin{aligned}
\frac{1}{a_{1}} \int_{\mathbb{R}^{N}} u^{q} v \phi^{2} d x \\
\quad=\frac{1}{a_{1}} \int_{\mathbb{R}^{N}} u^{(p-1) / 2} u^{(p+1) / 2 r} v \phi^{2} d x \\
=\frac{1}{a_{1}} \int_{\mathbb{R}^{N}} u^{(p-1) / 2} u^{(q+1) r /(r+1)} v \phi^{2} d x \\
\leq \frac{r}{r+1} \int_{\mathbb{R}^{N}} u^{(p-1) / 2} u^{q+1} \phi^{2} d x+\frac{1}{a_{1}^{r+1}(r+1)} \int_{\mathbb{R}^{N}} u^{(p-1) / 2} v^{r+1} \phi^{2} d x \\
=\frac{r}{r+1} I_{1}+\frac{1}{a_{1}^{r+1}(r+1)} I_{2},
\end{aligned}
$$

and similarly

$$
a_{2}^{r} \int_{\mathbb{R}^{N}} u^{p} v^{r} \phi^{2} d x \leq \frac{1}{r+1} I_{1}+\frac{a_{2}^{r+1} r}{r+1} I_{2} .
$$

Combining the above two inequalities and (3-8), we deduce that

$$
a_{2}^{r+1} I_{2} \leq\left(\frac{a_{2}^{r+1} r}{r+1}+\frac{1}{a_{1}^{r+1}(r+1)}\right) I_{2}+\frac{C}{R^{2}} \int_{B_{R_{k+1}}}\left(u^{q+1}+v^{r+1}\right) d x ;
$$

hence

$$
\frac{\left(a_{1} a_{2}\right)^{r+1}-1}{r+1} I_{2} \leq \frac{C a_{1}^{r+1}}{R^{2}} \int_{B_{R_{k+1}}}\left(u^{q+1}+v^{r+1}\right) d x .
$$

Thus, if $a_{1} a_{2}>1$, by the choice of $\phi$,

$$
\int_{B_{R_{k}}} u^{(p-1) / 2} v^{r+1} d x \leq I_{2} \leq \frac{C}{R^{2}} \int_{B_{R_{k+1}}}\left(u^{q+1}+v^{r+1}\right) d x .
$$

From (1-8) and (3-9), we get $u^{q+1} \leq C v^{r+1}$. Setting $s=r+1$, we can conclude that if $a_{1} a_{2}>1$,

$$
\begin{aligned}
\int_{B_{R_{k}}} u^{p} v^{s-1} d x \leq C_{1} \int_{B_{R_{k}}} u^{(p-1) / 2} v^{s} d x & \leq \frac{C_{2}}{R^{2}} \int_{B_{R_{k+1}}}\left(u^{q+1}+v^{r+1}\right) d x \\
& \leq \frac{C_{3}}{R^{2}} \int_{B_{R_{k+1}}} v^{s} d x .
\end{aligned}
$$

On the other hand, a simple verification shows that $a_{1} a_{2}>1$ is equivalent to $L(s)<0$. By Lemma 2.2, for $s \in\left[2 t_{0}, s_{0}\right)$, this last inequality holds. So the inequality (3-10), which is (3-1), holds for any $2 t_{0} \leq s<s_{0}$. On the other hand, the estimate (3-1) is valid for $2 \leq s<2 t_{0}$ [Cowan 2013, Lemma 4], hence for $2 \leq s<s_{0}$. 
We can then follow the iteration process in [Cowan 2013] (see Proposition 1 or Corollary 2 there) to obtain this consequence:

Corollary 3.2. Suppose $u$ is a classical stable solution of (1-1). For all $2 \leq \beta<$ $\frac{N}{N-2} s_{0}$, there are $\ell \in \mathbb{N}$ and $C<\infty$ such that

$$
\left(\int_{B_{R}} v^{\beta} d x\right)^{1 / \beta} \leq C R^{\frac{1}{2} N(2 / \beta-1)}\left(\int_{B_{R_{3}}} v^{2} d x\right)^{1 / 2} \text { for all } R>0 .
$$

Now we are in position to complete the proof of Theorem 1.1. Let $u$ be a smooth stable solution to (1-1). Corollary 3.2 and (2-1) imply that for any $2 \leq \beta<\frac{N}{N-2} s_{0}$, there exists $C>0$ such that

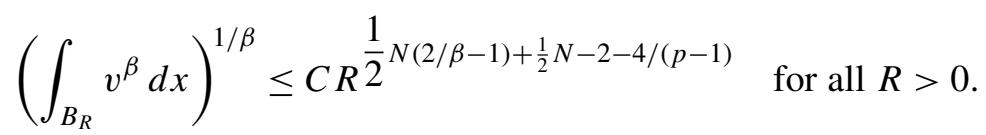

Note that

$$
\frac{1}{2} N(2 / \beta-1)+\frac{1}{2} N-2-\frac{4}{p-1}<0 \Longleftrightarrow N<\frac{2(p+1)}{p-1} \beta .
$$

Considering the allowable range of $\beta$ given in Corollary 3.2, if $N<2+\frac{2(p+1)}{p-1} s_{0}$, after sending $R \rightarrow \infty$ we get $\|v\|_{L^{\beta}\left(\mathbb{R}^{N}\right)}=0$, which is impossible since $v$ is positive. To conclude, the equation (1-1) has no classical stable solution if $N<2+2 x_{0}$ where $x_{0}=\frac{p+1}{p-1} s_{0}$.

Moreover, by Lemma 2.4, $x_{0}>5$ for any $p>1$, which means that if $N \leq 12$, (1-1) has no classical stable solution for all $p>1$.

\section{Proof of Theorem 1.4}

In this section, we consider the elliptic problem $\left(P_{\lambda}\right)$. Let $u_{\lambda}$ be the minimal solution of $\left(P_{\lambda}\right)$. It is well known that $u_{\lambda}$ is stable. To simplify the presentation, we erase the index $\lambda$. By [Cowan and Ghoussoub 2014; Dupaigne et al. 2013a],

$$
\sqrt{\lambda p} \int_{\Omega}(u+1)^{(p-1) / 2} \varphi^{2} d x \leq \int_{\Omega}|\nabla \varphi|^{2} d x \quad \text { for all } \varphi \in H_{0}^{1}(\Omega) .
$$

Using $\varphi=u^{(q+1) / 2}$ as a test function in (3-2), by similar computation as for (3-5) in Section 3, we obtain

$$
a_{1} \sqrt{\lambda} \int_{\Omega}(u+1)^{(p-1) / 2} u^{q+1} d x \leq \int_{\Omega} u^{q} v d x, \quad \text { where } a_{1}=\frac{4 q \sqrt{p}}{(q+1)^{2}} .
$$

Here we do not need a cutoff function $\phi$, because all boundary terms appearing in the integrations by parts vanish under the Navier boundary conditions, hence the 
calculations are even easier. We can use Young's inequality as for Theorem 1.1, but we show here a proof inspired by [Dupaigne et al. 2013b].

Similarly as for (3-7), using $\varphi=v^{(r+1) / 2}$ in (4-1), we have (4-3)

$$
a_{2} \sqrt{\lambda} \int_{\Omega}(u+1)^{(p-1) / 2} v^{r+1} d x \leq \int_{\Omega} \lambda(u+1)^{p} v^{r} d x, \quad \text { where } a_{2}=\frac{4 r \sqrt{p}}{(r+1)^{2}} .
$$

Fix $2 q=(p+1) r+p-1$. Applying Hölder's inequality,

$$
\begin{aligned}
\int_{\Omega} u^{q} v d x & \leq\left(\int_{\Omega} u^{(p-1) / 2} v^{r+1} d x\right)^{1 /(r+1)}\left(\int_{\Omega} u^{(p-1) / 2+q+1} d x\right)^{r /(r+1)} \\
& \leq\left(\int_{\Omega}(u+1)^{(p-1) / 2} v^{r+1} d x\right)^{1 /(r+1)}\left(\int_{\Omega} u^{(p-1) / 2+q+1} d x\right)^{r /(r+1)}
\end{aligned}
$$

and

$\int_{\Omega}(u+1)^{p} v^{r} d x \leq\left(\int_{\Omega}(u+1)^{(p-1) / 2} v^{r+1} d x\right)^{r /(r+1)}\left(\int_{\Omega}(u+1)^{(p-1) / 2+q+1} d x\right)^{1 /(r+1)}$.

Multiplying (4-2) with (4-3), using (4-4) and (4-5), we get immediately

$$
\left(\int_{\Omega}(u+1)^{(p-1) / 2} u^{q+1} d x\right)^{1 /(r+1)} \leq \frac{1}{a_{1} a_{2}}\left(\int_{\Omega}(u+1)^{(p-1) / 2+q+1} d x\right)^{1 /(r+1)}
$$

On the other hand, for any $\varepsilon>0$ there exists $C_{\varepsilon}>0$ such that

$$
(u+1)^{(p-1) / 2+q+1} \leq(1+\varepsilon)(u+1)^{(p-1) / 2} u^{q+1}+C_{\varepsilon} \quad \text { in } \mathbb{R}_{+} .
$$

If $a_{1} a_{2}>1$, there exists $\varepsilon_{0}>0$ satisfying $1+\varepsilon_{0}<\left(a_{1} a_{2}\right)^{r+1}$. We deduce from (4-6) that

$$
\left(1-\frac{1+\varepsilon_{0}}{\left(a_{1} a_{2}\right)^{r+1}}\right) \int_{\Omega}(u+1)^{(p-1) / 2} u^{q+1} d x \leq C .
$$

Therefore, when $L(s)<0$, or equivalently when $a_{1} a_{2}>1$, there is $C>0$ such that

$$
\int_{\Omega} u^{(p-1) / 2+q+1} d x \leq \int_{\Omega}(u+1)^{(p-1) / 2} u^{q+1} d x \leq C .
$$

Since $u^{*}=\lim _{\lambda \rightarrow \lambda^{*}} u_{\lambda}$, we conclude, using Lemma 2.2,

(4-7) $\quad u^{*} \in L^{(p-1) / 2+q+1}(\Omega) \quad$ for all $q$ satisfying $\frac{2(q+1)}{p+1}=r+1=s<s_{0}$.

Furthermore, by [Gazzola et al. 2010], we know that $u^{*} \in H^{2}(\Omega)$. Since $u^{*} \geq 0$ satisfies $\Delta^{2} u^{*}=\lambda^{*}\left(u^{*}+1\right)^{p} \leq C\left(u^{*}\right)^{p-1} u^{*}+C$ with $u^{*}=\Delta u^{*}=0$ on $\partial \Omega$, by 
standard elliptic estimate, we know that $u^{*}$ is smooth if

$$
\frac{N}{4}<\left(\frac{p-1}{2}+q+1\right) \frac{1}{p-1}=\frac{1}{2}\left(1+\frac{p+1}{p-1} s\right) .
$$

Therefore, $u^{*}$ is smooth if $N<2+2 x_{0}$. By Lemma 2.4, $u^{*}$ is smooth for any $p>1$ if $N \leq 12$.

\section{Acknowledgments}

Ye is partially supported by the French ANR project referenced ANR-08-BLAN0335-01. This work was partially realized during a visit of Harrabi to the University of Lorraine, and he would like to thank the Laboratoire de Mathématiques et Applications de Metz for the kind hospitality.

\section{References}

[Berchio and Gazzola 2005] E. Berchio and F. Gazzola, "Some remarks on biharmonic elliptic problems with positive, increasing and convex nonlinearities", Electron. J. Differential Equations (2005), Article ID \#34. MR 2006e:35067 Zbl 1129.35349

[Cowan 2013] C. Cowan, "Liouville theorems for stable Lane-Emden systems with biharmonic problems", Nonlinearity 26:8 (2013), 2357-2371. MR 3084715 Zbl 06203801

[Cowan and Ghoussoub 2014] C. Cowan and N. Ghoussoub, "Regularity of semi-stable solutions to fourth order nonlinear eigenvalue problems on general domains", Calc. Var. Partial Differential Equations 49:1-2 (2014), 291-305. MR 3148117

[Cowan et al. 2010] C. Cowan, P. Esposito, and N. Ghoussoub, "Regularity of extremal solutions in fourth order nonlinear eigenvalue problems on general domains", Discrete Contin. Dyn. Syst. 28:3 (2010), 1033-1050. MR 2011e:35085 Zbl 1196.35152

[Dupaigne et al. 2013a] L. Dupaigne, A. Farina, and B. Sirakov, "Regularity of the extremal solution for the Liouville system", pp. 139-144 in Geometric partial differential equations (Pisa, 2012), edited by A. Chambolle et al., CRM Series 15, Ed. Norm., Pisa, Pisa, 2013. MR 3156892 arXiv 1207.3703v1

[Dupaigne et al. 2013b] L. Dupaigne, M. Ghergu, O. Goubet, and G. Warnault, “The Gel'fand problem for the biharmonic operator", Arch. Ration. Mech. Anal. 208:3 (2013), 725-752. MR 3048594 Zbl 1284.35162

[Farina 2007] A. Farina, "On the classification of solutions of the Lane-Emden equation on unbounded domains of $\mathbb{R}^{N}$ ", J. Math. Pures Appl. (9) 87:5 (2007), 537-561. MR 2008c:35070 Zbl 1143.35041

[Ferrero et al. 2009] A. Ferrero, H.-C. Grunau, and P. Karageorgis, "Supercritical biharmonic equations with power-type nonlinearity", Ann. Mat. Pura Appl. (4) 188:1 (2009), 171-185. MR 2010g:35087 Zbl 1179.35125

[Gazzola and Grunau 2006] F. Gazzola and H.-C. Grunau, "Radial entire solutions for supercritical biharmonic equations”, Math. Ann. 334:4 (2006), 905-936. MR 2007b:35114 Zbl 1152.35034

[Gazzola et al. 2010] F. Gazzola, H.-C. Grunau, and G. Sweers, Polyharmonic boundary value problems: positivity preserving and nonlinear higher order elliptic equations in bounded domains, Lecture Notes in Mathematics 1991, Springer, Berlin, 2010. MR 2011h:35001 Zbl 1239.35002 
[Gui et al. 1992] C. Gui, W.-M. Ni, and X. Wang, "On the stability and instability of positive steady states of a semilinear heat equation in $\mathbb{R}^{n} "$, Comm. Pure Appl. Math. 45:9 (1992), 1153-1181. MR 93h:35095 Zbl 0811.35048

[Guo and Wei 2010] Z. Guo and J. Wei, "Qualitative properties of entire radial solutions for a biharmonic equation with supercritical nonlinearity", Proc. Amer. Math. Soc. 138:11 (2010), 39573964. MR 2012a:35054 Zbl 1203.35105

[Karageorgis 2009] P. Karageorgis, "Stability and intersection properties of solutions to the nonlinear biharmonic equation”, Nonlinearity 22:7 (2009), 1653-1661. MR 2011a:35240 Zbl 1172.31005

[Lin 1998] C.-S. Lin, "A classification of solutions of a conformally invariant fourth order equation in $\mathbb{R}^{n}$ ", Comment. Math. Helv. 73:2 (1998), 206-231. MR 99c:35062 Zbl 0933.35057

[Rellich 1969] F. Rellich, Perturbation theory of eigenvalue problems, Gordon and Breach, New York, 1969. MR 39 \#2014 Zbl 0181.42002

[Souplet 2009] P. Souplet, "The proof of the Lane-Emden conjecture in four space dimensions", $A d v$. Math. 221:5 (2009), 1409-1427. MR 2010h:35088 Zbl 1171.35035

[Wei and Xu 1999] J. Wei and X. Xu, "Classification of solutions of higher order conformally invariant equations”, Math. Ann. 313:2 (1999), 207-228. MR 2000a:58093 Zbl 0940.35082

[Wei and Ye 2013] J. Wei and D. Ye, "Liouville theorems for stable solutions of biharmonic problem", Math. Ann. 356:4 (2013), 1599-1612. MR 3072812 Zbl 06194421

[Wei et al. 2013] J. Wei, X. Xu, and W. Yang, "On the classification of stable solutions to biharmonic problems in large dimensions”, Pacific J. Math. 263:2 (2013), 495-512. MR 3068555 Zbl 06196725

Received March 1, 2013.

HATEM HAJLAOUI

InSTITUT DE MATHÉMATIQUES APPLIQUÉES ET D’INFORMATIQUES

3100 KAIROUAN

TUNISIA

hajlaouihatem@gmail.com

ABDELLAZIZ HARRABI

InSTITUT DE MATHÉMATIQUES APPLIQUÉES ET D’INFORMATIQUES

3100 KAIROUAN

TUNISIA

abdellaziz.harrabi@yahoo.fr

DONG YE

IECL, UMR 7502

UNIVERSITÉ DE LORRAINE

57045 METZ

FRANCE

dong.ye@univ-lorraine.fr 


\title{
PACIFIC JOURNAL OF MATHEMATICS
}

\author{
msp.org/pjm
}

Founded in 1951 by E. F. Beckenbach (1906-1982) and F. Wolf (1904-1989)

\section{EDITORS}

Don Blasius (Managing Editor)

Department of Mathematics

University of California

Los Angeles, CA 90095-1555

blasius@math.ucla.edu

\author{
Paul Balmer \\ Department of Mathematics \\ University of California \\ Los Angeles, CA 90095-1555 \\ balmer@math.ucla.edu \\ Robert Finn \\ Department of Mathematics \\ Stanford University \\ Stanford, CA 94305-2125 \\ finn@math.stanford.edu \\ Sorin Popa \\ Department of Mathematics \\ University of California \\ Los Angeles, CA 90095-1555 \\ popa@math.ucla.edu
}

\author{
Vyjayanthi Chari \\ Department of Mathematics \\ University of California \\ Riverside, CA 92521-0135 \\ chari@math.ucr.edu \\ Kefeng Liu \\ Department of Mathematics \\ University of California \\ Los Angeles, CA 90095-1555 \\ liu@math.ucla.edu \\ Jie Qing \\ Department of Mathematics \\ University of California \\ Santa Cruz, CA 95064 \\ qing@ cats.ucsc.edu
}

\section{PRODUCTION}

Silvio Levy, Scientific Editor, production@msp.org

\section{SUPPORTING INSTITUTIONS}

ACADEMIA SINICA, TAIPEI

CALIFORNIA INST. OF TECHNOLOGY

INST. DE MATEMÁTICA PURA E APLICADA

KEIO UNIVERSITY

MATH. SCIENCES RESEARCH INSTITUTE

NEW MEXICO STATE UNIV.

OREGON STATE UNIV.

\author{
STANFORD UNIVERSITY \\ UNIV. OF BRITISH COLUMBIA \\ UNIV. OF CALIFORNIA, BERKELEY \\ UNIV. OF CALIFORNIA, DAVIS \\ UNIV. OF CALIFORNIA, LOS ANGELES \\ UNIV. OF CALIFORNIA, RIVERSIDE \\ UNIV. OF CALIFORNIA, SAN DIEGO \\ UNIV. OF CALIF., SANTA BARBARA
}

\author{
Daryl Cooper \\ Department of Mathematics \\ University of California \\ Santa Barbara, CA 93106-3080 \\ cooper@math.ucsb.edu \\ Jiang-Hua Lu \\ Department of Mathematics \\ The University of Hong Kong \\ Pokfulam Rd., Hong Kong \\ jhlu@maths.hku.hk \\ Paul Yang \\ Department of Mathematics \\ Princeton University \\ Princeton NJ 08544-1000 \\ yang@math.princeton.edu
}

These supporting institutions contribute to the cost of publication of this Journal, but they are not owners or publishers and have no responsibility for its contents or policies.

See inside back cover or msp.org/pjm for submission instructions.

The subscription price for 2014 is US $\$ 410 /$ year for the electronic version, and \$535/year for print and electronic.

Subscriptions, requests for back issues and changes of subscribers address should be sent to Pacific Journal of Mathematics, P.O. Box 4163, Berkeley, CA 94704-0163, U.S.A. The Pacific Journal of Mathematics is indexed by Mathematical Reviews, Zentralblatt MATH, PASCAL CNRS Index, Referativnyi Zhurnal, Current Mathematical Publications and Web of Knowledge (Science Citation Index).

The Pacific Journal of Mathematics (ISSN 0030-8730) at the University of California, c/o Department of Mathematics, 798 Evans Hall \#3840, Berkeley, CA 94720-3840, is published twelve times a year. Periodical rate postage paid at Berkeley, CA 94704, and additional mailing offices. POSTMASTER: send address changes to Pacific Journal of Mathematics, P.O. Box 4163, Berkeley, CA 94704-0163.

PJM peer review and production are managed by EditFLOW ${ }^{\circledR}$ from Mathematical Sciences Publishers.

\section{PUBLISHED BY}

\section{mathematical sciences publishers \\ nonprofit scientific publishing}

http://msp.org/

(C) 2014 Mathematical Sciences Publishers 


\section{PACIFIC JOURNAL OF MATHEMATICS}

Volume $270 \quad$ No. $1 \quad$ July 2014

Hermitian categories, extension of scalars and systems of sesquilinear forms

EVA BAYER-FLUCKIGER, URIYA A. FIRST and DANIEL A.

MOLDOVAN

Realizations of the three-point Lie algebra $\mathfrak{s l}(2, \mathscr{R}) \oplus\left(\Omega_{\mathscr{R}} / d \mathscr{R}\right)$

BEN COX and ELIZABETH JURISICH

Multi-bump bound state solutions for the quasilinear Schrödinger equation with critical frequency

YUXIA GUO and ZHONGWEI TANG

On stable solutions of the biharmonic problem with polynomial growth

Hatem Hajlaoui, Abdellaziz Harrabi and Dong Ye

Valuative multiplier ideals

ZHENGYU HU

Quasiconformal conjugacy classes of parabolic isometries of complex hyperbolic space

YOUNGJU KIM

On the distributional Hessian of the distance function

Carlo MantegazZa, Giovanni Mascellani and Gennady URALTSEV

Noether's problem for abelian extensions of cyclic $p$-groups

IVO M. MICHAILOV

Legendrian $\theta$-graphs

Danielle O’Donnol and Elena PaVelescu

A class of Neumann problems arising in conformal geometry

WEIMIN SHENG and LI-XIA YUAN

Ryshkov domains of reductive algebraic groups

TAKAO WATANABE 